\title{
Absolute negative conductivity in two-dimensional electron systems under microwave radiation
}

\author{
Victor Ryzhii \\ Computer Solid State Physics Laboratory, University of Aizu, Aizu-Wakamatsu 965-8580, Japan
}

(Dated: June 13, 2018)

\begin{abstract}
We overview mechanisms of absolute negative conductivity in two-dimensional electron systems in a magnetic field irradiated with microwaves and provide plausible explanations of the features observed in recent experiments related to the so-called zero-resistance (zero-conductance) states.
\end{abstract}

PACS numbers: 73.40.-c, 78.67.-n, 73.43.-f

\section{INTRODUCTION}

The possibility of absolute negative conductivity (ANC) when the dc dissipative current is directed opposite to the local electric field in two-dimensional electron systems (2DESs) subjected to a magnetic field and irradiated with microwaves with the frequency $\Omega$ somewhat exceeding the cyclotron frequency $\Omega_{c}$ or its harmonics was predicted more than three decades ago [1] (see also Refs. 2, 3, 4, 5]). The predicted effect is associated with photon-assisted impurity scattering resulting in electron transitions between the states corresponding to different Landau levels (LLs) and different positions of the Larmor orbit centers. Two groups of experimentalists observed the effect of vanishing electrical resistance and transition to"zero-resistance" (in the Hall bar configuration) and "zero-conductance" (in the Corbino samples) states in 2DESs under microwave irradiation 6, 7, 8, 9]. Similar results were obtained also in Refs. 10, 11]. Recently, the oscillatory magnetic-field dependences of the Hall resistivity were observed 12, 13. The formation of zero-resistance and zero-conductance states is primarily attributed to photon-assisted impurity scattering mechanism of ANC 3, 4, 5, 14] and instability of uniform electric-field distributions due to ANC (for example, 15, 16]). In this paper, we address to the concept of ANC invoking the mechanism associated with the direct effect of microwave radiation on the electron scattering processes.

\section{PHASES OF OSCILLATIONS AND POWER EFFECTS}

The mechanism of ANC associated with photonassisted impurity scattering explains features of the effect. According to [1, 2, 3, 4], the positions of the photoconductivity zeros, maxima, and minima of the photoconductivity correspond mainly to $M \Omega=\Lambda \Omega_{c}$, $M \Omega=\Lambda \Omega_{c}-\delta^{(+)} \Omega_{c}$, and $M \Omega=\Lambda \Omega_{c}+\delta^{(-)} \Omega_{c}$, respectively, where $\Omega$ and $\Omega_{c}$ are the microwave and electron cyclotron frequencies, respectively, $M, \Lambda=1,2,3, \ldots$ and $\delta^{( \pm)}<1$ are determined either by the LL broadening $\Gamma$ or by the net electric field. Typical calculated dependences of the microwave photoconductivity corresponding to the

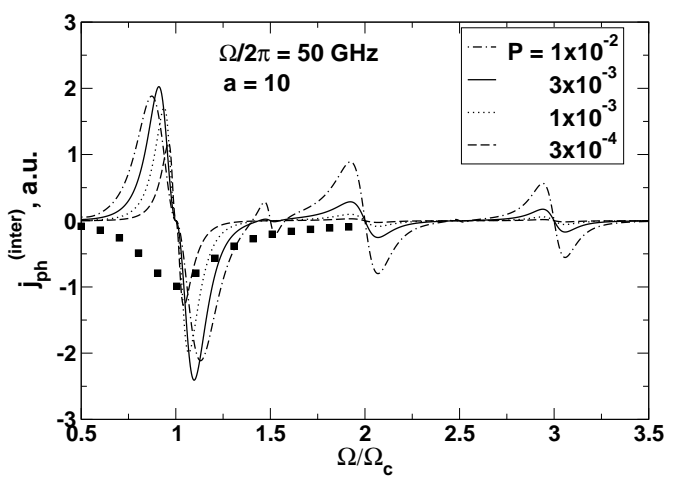

FIG. 1: Photocurrent due to photon-assisted impurity scattering vs $\Omega / \Omega_{c}$ at different microwave powers for $a=$ $\left(\Gamma / \Gamma_{i}\right)^{2}=10\left(\Gamma_{i}\right.$ is the LL broadening due to impurity scattering). Markers correspond to contribution of intra-LL electron transitions.

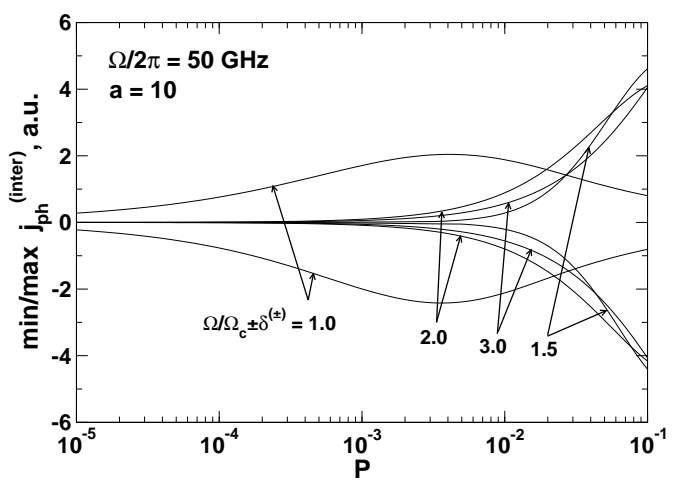

FIG. 2: Photocurrent maxima and minima corresponding to different resonances vs normalized microwave power.

abovementioned positions of the zeros, maxima, and minima are shown in Fig. 1 [4]. These dependences correlate well with the experimental curves. In particular, Fig. 1, shows a pronounced zero, maximum, and minimum near two-photon resonance $2 \Omega \simeq 3 \Omega_{c}$.

As seen from Fig. 1, the magnitude of the first maxi- 


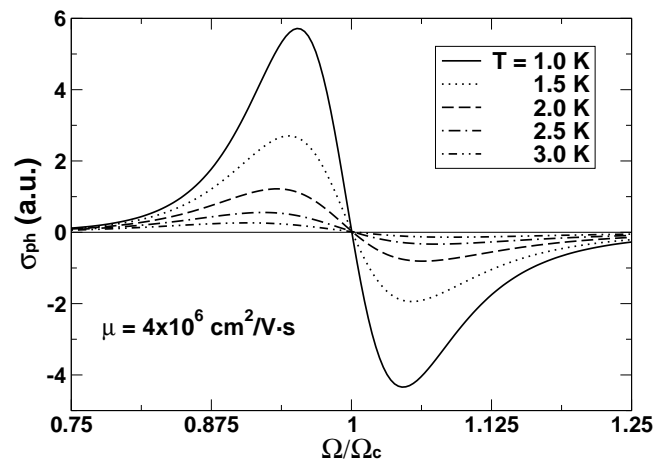

FIG. 3: Photoconductivity associated with photon-assisted impurity scattering vs $\Omega / \Omega_{c}$ at different temperatures.

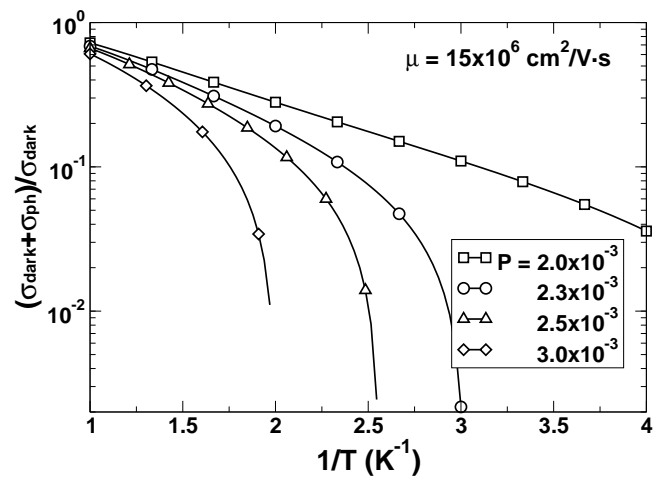

FIG. 4: Temperature dependences of normalized dissipative conductivity corresponding to different microwave powers.

mum and minimum $(\Lambda=1)$ as a function of microwave power tends to saturation when this power increases, while the span of the maxima and minima corresponding to higher resonances (see Fig. 2) continues to increase with increasing microwave power [4].

\section{TEMPERATURE SUPPRESSION OF ANC}

A significant sensitivity of the amplitude of microwave photoconductivity oscillations to the temperature can be attributed to an increase of the LL broadening due to increasing dependence of the electron-electron scattering. This is because the magnitude of the maxima and minima sharply decreases with increasing LL broadening. The microwave photoconductivity is actually determined by the processes of both absorption and emission of photons. This results in an additional temperature dependent factor: $\sigma_{p h} \propto[1-\exp (-\hbar \Omega / T)]$. At the photon energies $\hbar \Omega$ corresponding to the experimental values, this factor markedly decreases when the temperature increases from $T \lesssim 1 \mathrm{~K}$ to $T \simeq 3-5 \mathrm{~K}$. Considering this and assuming that the contribution of the electron-electron interaction to the LL broadening $\Gamma_{e} \propto T^{2} \ln \left(\varepsilon_{F} / T\right)$ [17, 18], where

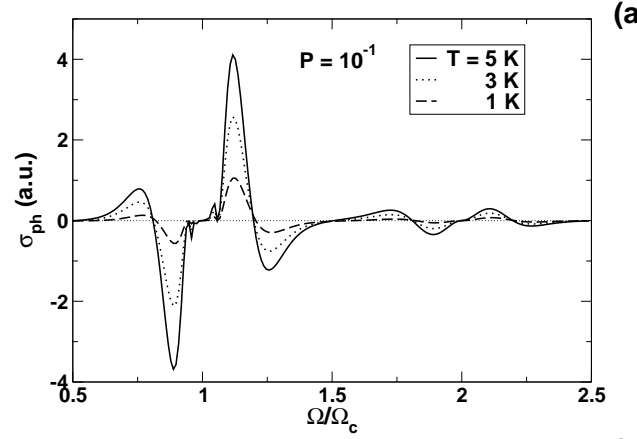

(a)

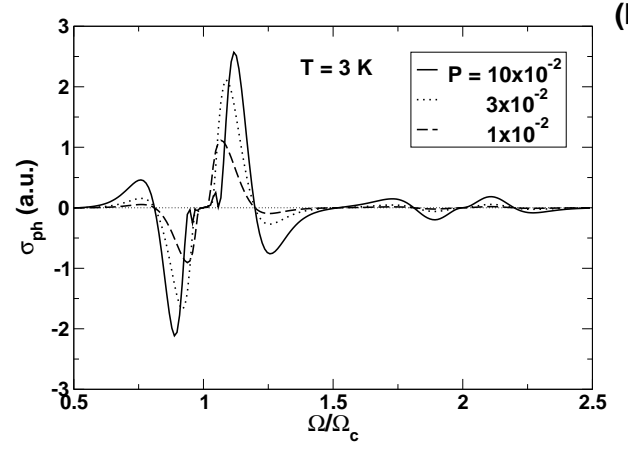

(b)

FIG. 5: Contribution of photon-assisted scattering on acoustic phonons to photoconductivity as a function of $\Omega / \Omega_{c}$ at different (a) temperatures and (b) microwave powers.

$T$ and $\varepsilon_{F}$ are the temperature and electron Fermi energy $\left(\varepsilon_{F} \gg \hbar \Omega_{c}, T\right)$, respectively, one can find that the shape of the first maximum and minimum transforms with temperature as shown in Fig. 3. Fig. 4 demonstrates the temperature dependences of the net dissipative conductivity at different microwave powers. Photonassisted scattering of electrons on acoustic phonons can also provide an oscillatory contribution to the microwave photoconductivity [19, 20]. The pertinent dependences calculated in [19] are shown in Fig. 5. As seen from Fig. 5, photon-assisted acoustic scattering leads to oscillatory microwave photoconductivity with the phase virtually opposite to the phase of the oscillation associated with photon-assisted impurity scattering. Since the contribution of the "phonon" mechanism increases with temperature, this mechanism can be weak at, for instance, $T \lesssim 1 \mathrm{~K}$ and be essential at elevated temperatures $(T \simeq 3-5 \mathrm{~K})$. The contribution of photon-assisted acoustic scattering to the microwave photoconductivity can be marked in 2DESs with rather high electron mobility, i.e., in the samples with sufficiently weak impurity scattering. As a result, the mechanism of ANC associated with photon-assisted acoustic scattering in 2DESs with very large mobilities can effectively interfere with the photon-assisted impurity scattering mechanism and surpass it even at fairly low temperatures. 


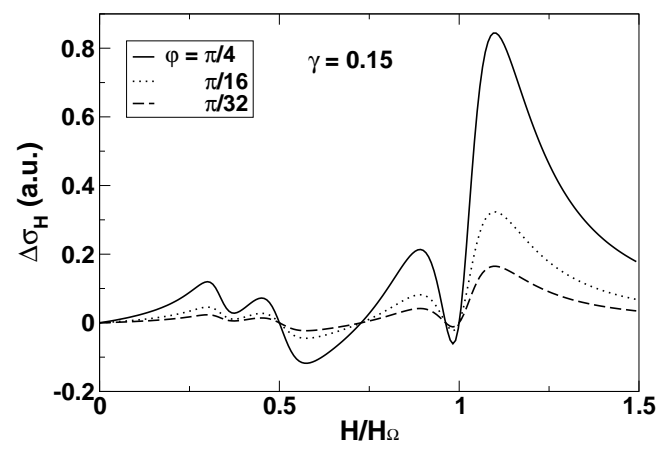

FIG. 6: The Hall microwave photoconductivity vs normalized magnetic field $\left(\gamma=\Gamma / \Omega_{c}=0.15\right)$.

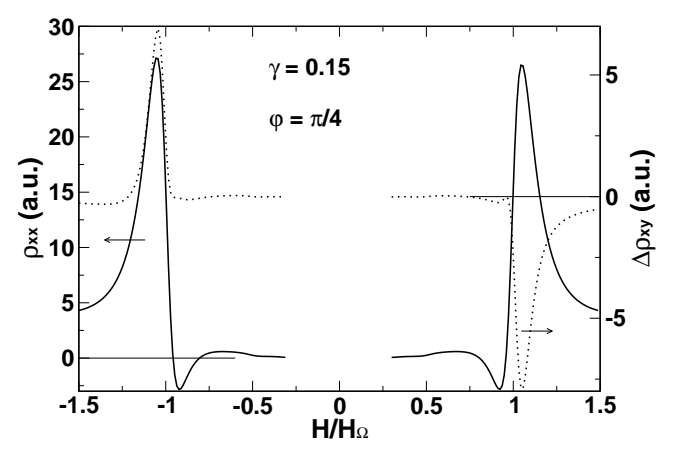

FIG. 7: Dissipative (solid curves) and Hall (dotted curves) resistivities vs normalized magnetic field.

\section{INFLUENCE OF MICROWAVES ON THE HALL EFFECT}

The photon-assisted impurity scattering provides contributions to both the dissipative and Hall components of the conductivity (and, consequently, resistivity) tensor. Fig. 6 shows the variation of the Hall conductivity under microwave radiation vs magnetic field normalized by $H_{\Omega} \propto \Omega$. It is instructive that the Hall microwave photoconductivity is an even function of the magnetic field. This component is sensitive to the polarization of microwave radiation. In the case of linear polarization, $\Delta \sigma_{H} \propto \sin 2 \varphi$ [21], as predicted a long time ago 22] (see also, [5] ), where $\varphi$ is the angle between the dc electric field and the ac microwave field. The dissipative and Hall microwave photoconductivities affect both the dissipative and Hall resistivities. Fig. 7 shows the calculated dissipative resistivity and variation of the Hall resistivity as functions of the normalized magnetic field [22]. These dependences are similar to the experimental one's [12, 13].

\section{COMMENTS}

Apart from the mechanisms of ANC associated with the effect of the microwave field on the electron dynamics and scattering processes, the effect of ANC in a 2DES subjected to a magnetic field can also be associated with a deviation of the electron distribution function from equilibrium one ("statistical" mechanism). A model based on the assumption that the electron distribution function is nonmonotonic with inversion population of the states near the center of LL's caused by the absorption of microwave radiation was proposed [11, 23]. In this model, a photoexcited electron contributes to the dissipative photoconductivity immediately when it transfers to an upper LL absorbing a photon (this actually corresponds to the dynamic mechanism) and owing to non-radiative scattering events upon the excitation. The impurity scattering of the photoexcited electrons can provide a negative contribution to the dissipative photoconductivity if the maxima of the electron density of states are shifted with respect to the maxima of the distribution function [24]. The main problem is, however, the feasibility of such nontrivial electron distributions, particularly, due to both intra-LL and inter-LL electron-electron scattering 18, 25] and scattering of electrons on acoustic phonons [26]. The dynamical and statistical mechanisms can be distinguished by their different polarization selectivity.

In summary, we demonstrated that the main features of the mechanism of ANC associated with photonassisted scattering of electrons on impurities and acoustic phonons are consistent with the results of experimental observations.

The author is grateful to R. Suris, V. Volkov, A. Chaplik, and V. Vyurkov for discussions and to I. Khmyrova and A. Satou for assistance.
[*] Electronic address: v-ryzhii@u-aizu.ac.jp

[1] V. I. Ryzhii, Sov. Phys.-Solid State 11, 2078 (1970).

[2] V. I. Ryzhii, R. A. Suris, and B. S. Shchamkhalova, Sov. Phys.-Semicond. 20, 1299 (1986); Physica E (2004), in press.

[3] A. C. Durst, S. Sachdev, N. Read, and S. M. Girvin, Phys. Rev. Lett. 91, 086803 (2003).

[4] V. Ryzhii and R. Suris, J. Phys.: Cond. Mat. 15, 6855 (2003).

[5] M. G. Vavilov and I. L. Aleiner, Phys. Rev. B 69, 035303
(2004).

[6] R. G. Mani, J. H. Smet, K. von Klitzing, V. Narayanamurti, W. B. Johnson, and V. Umansky, Nature 420, 646 (2002).

[7] M. A. Zudov, R. R. Du, L. N. Pfeiffer, and K. W. West, Phys. Rev. Lett. 90, 046807 (2003).

[8] C. L. Yang, M. A. Zudov, T. A. Knuuttila, R. R. Du, L. N. Pfeiffer, and K. W. West, Phys. Rev. Lett. 91, 096803 (2003).

[9] R. G. Mani, J. H. Smet, K. von Klitzing, 
V. Narayanamurti, W. B. Johnson, and V. Umansky, cond-mat/0306388 (2003).

[10] R. L. Willett, L. N.Pfeiffer, and K. W. West, cond-mat/0308406 (2003).

[11] S. I. Dorozhkin, JETP Lett. 77 (2003) 681.

[12] S. A. Studenikin, M. Potemski, P. T. Coleridge, A. Sachrajda, and Z. R. Wasilewski, cond-mat/0310347 (2003).

[13] R. G. Mani, J. H. Smet, K. von Klitzing, V. Narayanamurti, W. B. Johnson, and V. Umansky, cond-mat/0310474 (2003).

[14] P. W. Anderson and W. F. Brinkman, cond-mat/0302129 (2003).

[15] A. V. Andreev, I. L. Aleiner, and A. J. Millis, Phys. Rev. Lett. 91, 056803 (2003).

[16] F. S. Bergeret, B. Huckestein and A . F. Volkov, Phys. Rev. B 67, 241303 (2003).
[17] A. V. Chaplik, Sov. Phys. JETP 33, 997 (1971).

[18] Y. Levinson, Phys. Rev. B 51, 16898 (1995).

[19] V. Ryzhii and V. Vyurkov, Phys. Rev. B 68, 165406 (2003).

[20] V. Ryzhii, Phys. Rev. B 68, 193402 (2003).

[21] V. Ryzhii, cond-mat/0310757 (2003); J. Phys. Soc. Japan, in press.

[22] A. D. Gladun, A. D. Malov, and V. I. Ryzhii, JETP Lett. 21, 2 (1975).

[23] I. A. Dmitiev, M. G. Vavilov, I. L. Aleiner, A. D. Mirlin, and D. G. Polykov, cond-mat/0310668 (2003).

[24] V. F. Elesin, Sov. Phys. JETP 28, 410 (1969).

[25] E. Tsitsishvili and Y. Levinson, Phys. Rev. B 56, 6921 (1997).

[26] S. Iordanski and Y. Levinson, Phys. Rev. B 53, 7308 (1996). 